\title{
Relevant Independent Variables on MOBA Video Games to Train Machine Learning Algorithms
}

\author{
Juan Guillermo López Guzmán \\ Pontifical Xaverian University \\ 110121, Bogotá, Bogotá DC \\ juang.lopezg@javeriana.edu.co
}

\author{
Cesar Julio Bustacara Medina \\ Pontifical Xaverian University \\ 110121, Bogotá, Bogotá DC \\ cbustaca@javeriana.edu.co
}

\begin{abstract}
Popularity of Multiplayer Online Battle Arena (MOBA) video games has grown considerably, its popularity as well as the complexity of their playability, have attracted the attention in recent years of researchers from various areas of knowledge and in particular how they have resorted to different machine learning techniques. The papers reviewed mainly look for patterns in multidimensional data sets. Furthermore, these previous researches do not present a way to select the independent variables (predictors) to train the models. For this reason, this paper proposes a list of variables based on the techniques used and the objectives of the research. It allows to provide a set of variables to find patterns applied in MOBA videogames. In order to get the mentioned list, the consulted works were grouped by the used machine learning techniques, ranging from rule-based systems to complex neural network architectures. Also, a grouping technique is applied based on the objective of each research proposed.
\end{abstract}

\section{Keywords}

Pattern Recognition, recommender systems, Machine Learning, MOBA, League of Legends.

\section{INTRODUCTION}

Videogames are already present in our everyday life. They have reached a massive audience from all ages due to their wide variety of genders and thematics. Those genders evolve trought time and originate new ones, for instance, Real Time Strategy games (RTS) originates at the early 2000's a different sub-gender known as Massive Online Battle Arena (MOBA) [1].

There are many games that could be considered as MOBA predecessors. Although, the first videogame formally classified as a MOBA was Defence of the Ancients (DotA). It was created in 2003 by players of the RPG videogame Warcraft III. DotA is based on the Warcraft III lore, however, it has specific gameplay characteristics. These characteristics have become intrinsic to MOBA gender:

Each match starts with two adversary teams, typically conformed of five players. Players work together as a team to achieve the ultimate victory condition which is

Permission to make digital or hard copies of all or part of this work for personal or classroom use is granted without fee provided that copies are not made or distributed for profit or commercial advantage and that copies bear this notice and the full citation on the first page. To copy otherwise, or republish, to post on servers or to redistribute to lists, requires prior specific permission and/or a fee. to destroy their enemy's base whilst protecting their own [1]. Usually, both teams have main structures that are located at the opposite corners of the battlefield. The first team to destroy the adversaries' main structure wins the match [1]. Destroying other structures within the adversary team's base may provide other benefits. Defensive structures, which are usually automatic "towers", are located in place to prevent the base destruction. Each team is assisted by relatively weak computer-controlled units, called "minions", that periodically spawn in groups at both bases, marching down predefined paths (called "lanes") toward their enemy base. While their charges are counterbalanced by the adversary team's minions. Players can aid them which turns the minions into a useful army for striking the adversaries' defenses [2]. There are typically three (3) "lanes" on the battlefield that are the main ways of getting from one base to another. The lanes are known as top, middle and bottom lane, or, in gamer shorthand - "top", "mid" and "bot". Between the lanes is an uncharted area called "jungle" [3]. The "jungle" is a territory to neutral monsters that are hostile to both teams and appear in marked locations on the map known as "camps" [4]. Defeating neutral monsters brings various benefits to the players and their team, such as growth in power, buffs, or assistance in pushing the lane [5].

In 2009 and 2012 respectively born the most popular MOBA videogames: League of Legends (LoL) and 
Defence of the Ancients II (DotA II). They maintain the gameplay described above: Two teams of five players have to destroy the nexus of the enemy team located in the different side of a predefined map. Fighting against defensive structures, player and non-player characters.

Both achieved great popularity in the e-sports scenario. For instance, 2019 League of Legends World Championship got an online audience greater than one hundred million through several electronic platforms as Twitch [7]. Also, it got an in-site audience near to forty thousand people on the Shanghai Olympic stadium.

Due to players face several real-time decisions with a wide spectrum of possibilities MOBAs are very complex videogames [8]. Mentioned complexity and their popularity have attracted a lot of researchers' attention from various knowledge fields as psycology, marketing, computer science and artificial intelligence. Figure 1 shows the growth in the number of reviewed papers by year as evidence of the researchers' interest since 2014.

The above complexity had allowed the application of a wide spectrum of machine learning techniques from regression models [6] to different neural networks architectures [7]. Technique's evolution will be presented in Section 2.

There are several objectives related to MOBA's research. For instance: how game dynamics differentiate one player from another [7], how the chosen avatar has impact in the odds to win a game [8]. Every found objective will be described in Section 3.

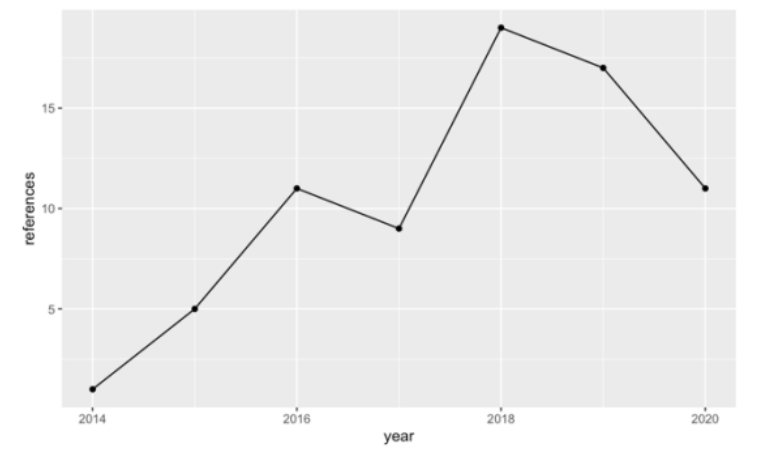

Figure 1. Number of reviewed references per year.

One important decision is how to define what items a player should buy in order to maximize his chance to win a game. As mentioned above, every decision in a MOBA videogame has a wide spectrum of feasible solutions turning them into complex optimization problem. Each player could buy until six items in each game from a pool higher than eighty options, for that reason, the number of possible builds is around $2.2 \mathrm{e} 11$ combinations.
According to the number of possible decisions that players can take, there are many variables to consider. In consequence the present work contains the sections describe below: Section 2 describes the most common machine learning techniques used on MOBA videogames with a special emphasys on what independent variables have been used in each technique. Section 3 groups the consulted researches by his objective and what independent variables have been used in each one. Section 4 presents a summary of the results and introduce the proposed list of relevant variables. Finally, Section 5 has main conclussiones and describes future works.

\section{MOBA'S MACHINE LEARNING TECHNIQUES LISTING}

An exhaustive research was performed in several academical repositories to collect researches related to MOBA videogames. Researches where investigators have used a machine learning technique was reviewed in detail to identify what techniques they were using and, the question investigators were trying to answer within the research.

Every reviewed paper uses one or more techniques to solve its objective. These techniques were tabulated and clustered into categories. It helped to find the most popular technique categories: Heuristics, neural networks, regression models and trees-based models. Figure 2 shows their evolution through time.

Most popular technique categories will be described in further sub-sections. As mentioned before, for each category will be listed the independent variables used by. Finally, there will be a sub-section to describe the methods and independent variables used in other categories.

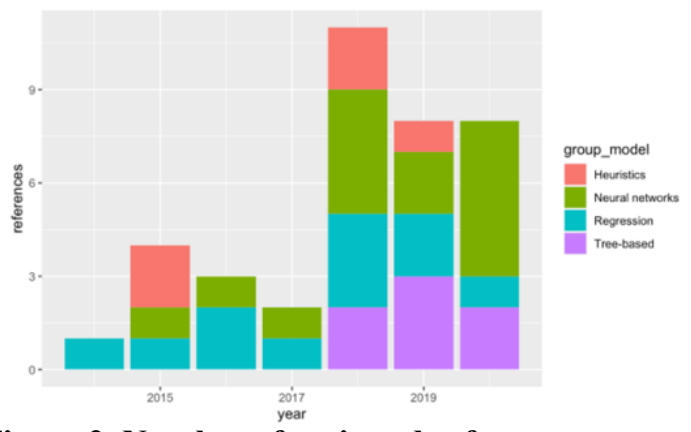

Figure 2. Number of reviewed references grouped per year and technique category.

\subsection{Heuristics}

During early years, heuristics were the predominant models which mainly use rule-based systems. For instance, a decisions' tree that will describe a rational agent who works like a novice players tutor [9] which 
provide a rational agent the actions to execute whether the human player are dead, absent or under attack.

This category also contains: To develop an Artificial Intelligence MOBA player [10], adjusting the difficulty of a rational agent based on player performance [11], to suggest the avatar to choose in order to maximize the odds to win a game [8] [12] and which items should be bought during a game [13].

Table 1 presents the independent variables used as predictors in heuristic models and their number of appearances in different papers.

\begin{tabular}{llr}
\hline \multicolumn{1}{c}{ variable } & type & $\begin{array}{c}\text { number of } \\
\text { appearances }\end{array}$ \\
\hline Attacked by tower & boolean & 1 \\
Champion range & float & 1 \\
Creep missing percentage of health points & float & 1 \\
Distance between champions and creep & float & 1 \\
Distance between champions and tower & float & 1 \\
In turret & boolean & 1 \\
Items & vector & 1 \\
Partner AFK & boolean & 1 \\
Partner alive & boolean & 1 \\
Partner close & boolean & 1 \\
Partner dead & boolean & 1 \\
Partner recalling & boolean & 1 \\
Safe in ally turret & boolean & 1 \\
Teammate around & boolean & 1 \\
Tower range & float & 1 \\
\hline
\end{tabular}

Table 1. Independent variables used in Heuristics Models.

\subsection{Regression Models}

Another relevant category during early years is regression models. They were applied mainly to predict which team will win a game. Then, as a classification problem the more used method has been logistic regression [6] [14] [15] [16] [17]. Regression models have also been used to explain how the way players and teams move influences the game result [18] or how game result is influenced by bought items [19]. Finally, researchers have used generalized linear models to model the performance on professional teams during the 2018 League of Legends world championship [20].

Regression models have used 65 different independent variables. Those that were used in more than one model were shown in Table 2.

\subsection{Tree-Based Models}

A recently popular category has been tree-based models as simple classification trees [21] and behavior trees [22]. However, most used methodology has been random forest:

- to predict the learning ratio for a new player [16].

- to determine whether a skill or a usable item is available to be used [23].
- $\quad$ to predict the game outcome [24] [25] and

- to suggest the avatar to be used into a game [17] [26].

They have used 38 independent variables. Table 3 shows the variables that have been used into more than one model.

\begin{tabular}{llr}
\hline \multicolumn{1}{c}{ variable } & type & $\begin{array}{c}\text { number of } \\
\text { appearances }\end{array}$ \\
\hline Chosen hero & vector & 4 \\
Gold & integer & 4 \\
Creeps & integer & 3 \\
Assists & integer & 2 \\
Damage to heroes & integer & 2 \\
Deaths & integer & 2 \\
Gold difference between teams & integer & 2 \\
Items & vector & 2 \\
KDA Ratio & float & 2 \\
Kills & integer & 2 \\
Roles & vector & 2 \\
\hline
\end{tabular}

Table 2. Independent variables used in Regression Models.

\begin{tabular}{llr}
\hline \multicolumn{1}{c}{ variable } & type & $\begin{array}{r}\text { number of } \\
\text { appearances }\end{array}$ \\
\hline Chosen hero & vector & 3 \\
Kills & integer & 3 \\
Bans & vector & 2 \\
Creeps & integer & 2 \\
Gold difference between teams & integer & 2 \\
Roles & vector & 2 \\
\hline
\end{tabular}

Table 3. Independent variables used in Based-Tree Models.

\subsection{Neural Networks}

Recently, neural networks had become the predominant techniques during the last years. The variety of architectures in this group is remarkable. There could be found simple artificial neural networks [7] [27] [28] [19] [29], or more complex architectures: recurrent neural networks [30] [31] [32], convolutional neural networks [23], deep neural networks [33] [34] [35], fully-connected neural networks [26] [35], discriminative neural networks [36] and transformers [37].

Into the reviewed papers which used a neural network model were identified 42 independent variables. Table 4 presents those used into more than one paper.

\begin{tabular}{llr}
\hline \multicolumn{1}{c}{ variable } & type & $\begin{array}{c}\text { number of } \\
\text { appearances }\end{array}$ \\
\hline Chosen hero & vector & 4 \\
Items & vector & 3 \\
Gold & integer & 2 \\
Gold difference between teams & integer & 2 \\
Kills difference between teams & integer & 2 \\
Tower kills difference between teams & integer & 2 \\
\hline
\end{tabular}

Table 4. Independent variables used in Neural Network Models. 


\subsection{Other Techniques}

In addition to the most popular categories, there are reviewed papers focused on to develop visualization methods to improve the comprehension about game patterns. They could be used by players [38] [39], coaches [40] [41] [42] [43] and spectators [44].

Also, classification models based on linear hyperplanes where support vector machines are the most popular [6] [45] [23] [17] accompanied by factorial [15].

Other categories are: Boosting [15] [46] [24] [17], clusterization [18] [47] [13] [29], Bayesians [6] [30] [15], behavioral [7] [48] [49] [50], bio-inspired [45] [51] [52], network analysis [53] [43] [54], mixed models [55] [56], recommender system [57] [27], fuzzy logic [58] [59], singular value decomposition [60], auto-regressive [61] and sequence analysis [50].

Summarizing the reviewed researches it is possible to notice: a) the intrinsic complexity of MOBA videogames let researchers to use a wide spectrum of machine learning techniques and $b$ ) there are variables which are repeated transversally in several researches, even when the used tecniques are evolve through time there are variables like the chosen hero present in three of the four tables shown.

\section{ObJective CATEgories}

As previous section clustered the reviewed papers into categories based on the applied machine learning techniques, this section will tabulate and cluster researches based in the paper objectives. Figure 3 describes ths most popular categories.

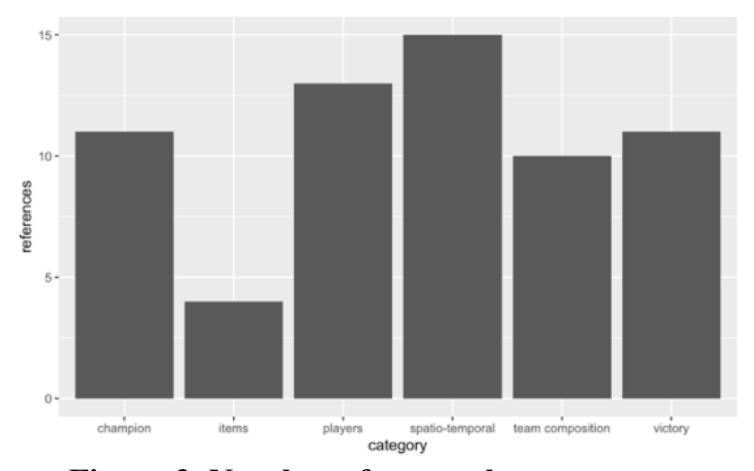

Figure 3. Number of researches per category.

Each category will be explored in detail during the further sub-sections. Besides, aligned with the main goal for each category will be analyzed the used independent variables.

\subsection{Spatio-Temporal}

Most popular category was called spatio-temporal. It refers to researches that have sought patterns related to how the game is developed in different map coordinates or different time slices:

- To detect an experienced player based in the way that he navigates across the game map [18] [39] [41] [43].

- To develop rational agents which determine the best way to navigate the game map at a specific moment of the game [9] [10] [35].

- To analyze the advantage of one of the teams at a specific time during the game [28] and specifically when this advantage generates an irreversible snowball effect [38].

- To improve spectators experience by determining in which sectors of the game map the events most relevant to the game are taking place [46] [55].

- To predict the outcome of an encounter with two or more players [14] [61] [34].

- Finally, to determine whether an ability or an actionable item could be used [23].

Although, it was possible to identify 64 different independent variables that were used in the aforementioned research. Due to the variety of topics, only 3 were used in more than one work, these are presented in Table 5.

\begin{tabular}{llr}
\hline \multicolumn{1}{c}{ variable } & type & $\begin{array}{c}\text { number of } \\
\text { appearances }\end{array}$ \\
\hline Gold difference between teams & integer & 3 \\
Experience difference between teams & integer & 2 \\
Positions of avatars & tuples & 2 \\
\hline
\end{tabular}

Table 5. Independent variables used in SpatioTemporal Research.

\subsection{Players}

The second category was called players. Players' category groups the research that analyze characteristics of the players. These characteristics do not depend on the game condition, on the contrary, they tend to be present into every player's game. In this category we found objectives such as:

- To adjust automatically the level of difficulty for any player based on his historical performance [58].

- To predict the outcome of a game based on the historical performance of the players [62] [31] [50]

- To categorizing players according to their historical performance and design analysis strategies to improve it [40] [41] [42] [43] [29].

- To analyze the learning speed of a new player [16].

- To predict based on their gaming habits if a player is at risk of quitting the game [48], the best way to match players based on their 
historical performance [33] [63] and,

- how the player's social network within the video game affects the outcome of his games [53].

For this category, a total of 51 different independent variables were identified. Table 6 shows those used in more than one research.

\begin{tabular}{llr}
\hline variable & type & $\begin{array}{r}\text { number of } \\
\text { appearances }\end{array}$ \\
\hline Gold & integer & 5 \\
Creeps & integer & 3 \\
Assits & integer & 2 \\
Deaths & integer & 2 \\
Items & vector & 2 \\
KDA Ratio & float & 2 \\
Kills & integer & 2 \\
Roles & vector & 2 \\
Start time & timestamp & 2 \\
Winning & boolean & 2 \\
\hline
\end{tabular}

Table 6. Independent variables used in Players Research.

\subsection{Champions}

The researches that study the impact of the avatar selected by the players have been grouped into the champions category. Due to it is the way in which avatars are known within the MOBA League of Legends. The main objective in these researches has been to design champion recommendation systems [30] [27] [8] [12] [51] [17] [64] [60] [36] [26] [32] and predict the outcome of a game [15] [45] [7].

Although these works use a total of 18 different independent variables, the only one that appears in all researches is 'chosen-hero': a vector that represents the champion selected by one or more players.

\subsection{Victory}

Another of the objectives found in selected researches is the prediction of the winning team in a game. This category uses variables that are known at the beginning of the game [15] [27] [60] [43] [31] [65] [24] [25], at any time during the game [14] [62] or variables known at the end of a game in order to understand their influence on the result [66] [54] [20]. Therefore, this category will be called victory.

Of the 49 independent variables used within this category, table 7 shows those that were used in more than one research.

\subsection{Team Composition}

A limitation commonly mentioned in research is how to introduce into the models the synergies that must exist between players of the same team. Related to this question the category of team composition was created for those papers that seek objectives related to the roles of each player within a team [6] [50], the analysis of player behavior in the composition of a team [7] [45] [67] [42] [68], predicting the outcome of a game considering synergies between players [15] [65] or optimizing the construction of a team of players [8] [32] [49] [52] [63].

\begin{tabular}{llr}
\hline \multicolumn{1}{c}{ variable } & \multicolumn{1}{c}{ type } & $\begin{array}{c}\text { number of } \\
\text { appearances }\end{array}$ \\
\hline Chosen hero & vector & 5 \\
Gold & integer & 3 \\
Gold difference between teams & integer & 3 \\
Creeps & integer & 2 \\
Creeps per minute & float & 2 \\
Gold per minute & float & 2 \\
Kills & integer & 2 \\
Level & integer & 2 \\
Roles & vector & 2 \\
\hline
\end{tabular}

Table 7. Independent variables used in Victory Research.

A total of 45 different independent variables were identified in the works within this category. Table 8 presents those used in more than one research.

\begin{tabular}{lrr}
\hline \multicolumn{1}{c}{ variable } & \multicolumn{1}{c}{ type } & $\begin{array}{c}\text { number of } \\
\text { appareances }\end{array}$ \\
\hline Assists & integer & 2 \\
Chosen hero & vector & 2 \\
Creeps & integer & 2 \\
Kills & integer & 2 \\
\hline
\end{tabular}

Table 8. Independent variables used in Team Composition Research.

\subsection{Items}

One of the least explored dimensions in the game is the purchase of items to equip each player's virtual avatar. In League of Legends, each player has the possibility of acquiring up to six objects throughout a game from a pool of more than 80 different objects, all with different benefits for the character. The researches that study this game characteristic were grouped within the category objects. These seek to develop systems that recommend to the player which are the objects to acquire during the game [13] [69] [19] [35].

In a similar way as in the champions category, the only variable that appears more than once in the item's category is 'items': a binary vector that identifies whether or not a champion has a specific item equipped.

It is important to mention that none of the researches in the item's category considers the player's resources when is generated a recommendation. It could be obsolete if player does not have enough gold to acquire 
the suggested item.

\section{Analysis of Results}

In total, 165 different independent variables were identified. This number is a consequence of the variety of objectives identified in the reviewed researches, therefore, many of these variables are used only in one paper or one objective category.

The variables aggrupation presented could be useful for future researches, specifically, authors future work will be focus on: how to define the items that maximize the chance to win a game. In consequence, results related with the categories players, champions and items will be highlighted. These categories were selected based on the qualitative hypothesis described below:

i. Players: These researches describe characteristics and play styles that are present in every player's games. It indicates that there may be a basket of items a player acquires in every game.

ii. Champions: Each virtual avatar has different characteristics (speed, armor, attack speed, ability power, among others) just like each object, therefore, each champion can have a greater affinity with those objects that share similar characteristics.

iii. Items: The papers within this category share the objective of future work.

The set of independent variables identified (165) were mapped into the three chosen categories, as presented in Figure 4 only three variables are repeated in more than one category.

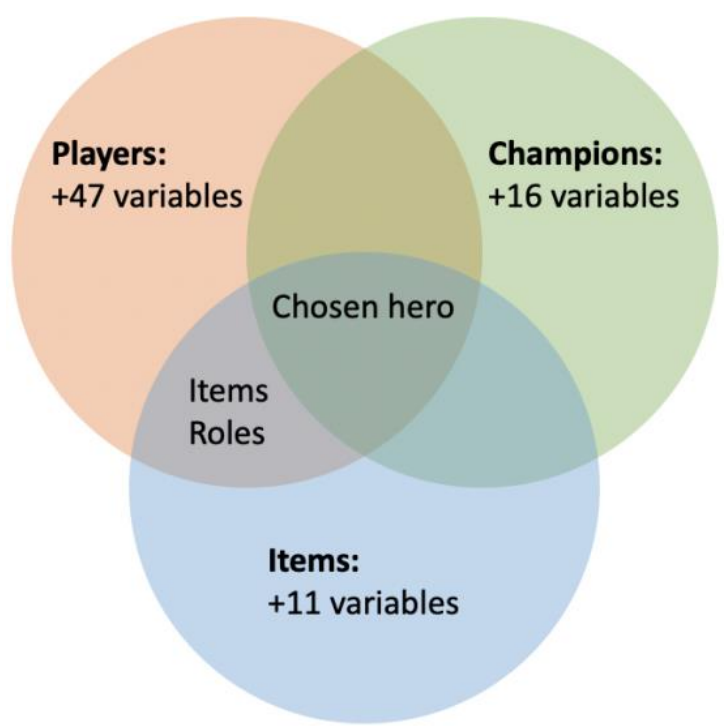

Figure 4. Independent variables that are repeated throughout the categories of interests. 'chosen hero' variable is a binary vector that represents whether a player or a team has selected a specific champion. 'items' variable is also a binary vector that indicates whether a player or a team has equipped their champion with a specific object. 'roles' is a categorical variable that indicates the role of each player in the game.

As evidenced in [70], in order to keep the champions balanced, the developer company makes adjustments to champion and item attributes, it happens in the same way with the objects, for that reason, the relations and synergies in 'chosen hero' and 'items' variables are constantly changin. Therefore, using these vectors of binary variables as predictors could quickly become obsolete after an update. However, both variables can be expressed based on the attributes of champions and items, so these predictors will not be linked to a specific champion / item with specific characteristics but to any champion / item whose attributes are similar to the vector of attributes trained by the model.

\section{Conclusions}

Thanks to the exhaustive papers review, built categories and variables match, presented in previous sections, it is possible to conclude:

i. Reviewed papers do not show a consistency in the independent variables use, even in researches with related objectives. It suggests variables were mainly chosen by the authors preferences and it is not related to previous researches or a specific methodology.

ii. It is possible to determine the chosen hero, the items and roles are the most commonly used variables and, for that reason, they could be considered as a baseline variables to machine learning applications in MOBA videogames.

Author's future work will be focused on the importance of bought items in the victory probability during a match. Below the acquire lessons that will be useful:

i. Variables mentioned (chosen hero, items and roles) will be used as baseline predictors to train machine learning models to determine the impact of items in the probability to win a match.

ii. Due to the periodic changes in the champion and items stats [71] future works will suggest representing the independent variables (chosen hero and items) in a way agnostic to the current game patch. So far, they were represented as binary vectors, in consequence, the stats updates made trained models less relevant with every patch. 
iii.

\begin{abstract}
None of the researches reviewed consider into their models the player available resources, it means, suggestions made are optimal ones considering a player can buy any item he wants in any time during the game. Then, considering available resources when suggesting an item will be an exclusive contribution of future works.
\end{abstract}

\section{REFERENCES}

[1] L. Jackson, "How MOBAs Took Over Gaming," IGN Middle East, 1 August 2013. [Online]. Available: https://me.ign.com/en/pc/70142/feature/how-mobastook-over-gaming. [Accessed 23 March 2021].

[2] Field Level Media, "Esports primer: League of Legends," Reuters, 24 March 2020. [Online]. Available: https://www.reuters.com/article/esports-lol-primeridUSFLM1tj2jd. [Accessed 23 March 2021].

[3] J. Calixto, "Proving Grounds: The Geography of the MOBA Map," The Meta, [Online]. Available: https://killscreen.com/themeta/proving-groundsgeography-moba-map/. [Accessed 23 March 2021].

[4] A. Gies, "The Normal Person's Guide to Watching Competitive Dota 2 (2017 Edition)," Polygon, 2 August $2017 . \quad$ [Online]. Available: https://www.polygon.com/2017/8/2/16073588/thenormal-persons-guide-to-watching-competitive-dota-22017-edition. [Accessed 23 March 2021].

[5] Garamor, "Heroes of the Storm: How to Fully Utilize Mercenary Camps," Dignitas, 27 October 2017. [Online]. Available: http://teamdignitas.net/articles/blogs/Heroes-Of-TheStorm/11768/heroes-of-the-storm-how-to-fully-utilizemercenary-camps. [Accessed 23 March 2021].

[6] C. Eggert, M. Herrlich, J. Smeddinck and R. Malaka, "Classification of player roles in the team-based multiplayer game dota 2," in International Conference on Entertainment Computing, 2015.

[7] H. Wang, H.-T. Yang and C.-T. Sun, " Thinking style and team competition game performance and enjoyment," IEEE Transactions on Computational Intelligence and A/ in Games, vol. 7, no. 3, pp. 243-254, 2015.

[8] Z. Chen, T.-H. D. Nguyen, Y. Xu, C. Amato, S. Cooper, Y. Sun and M. S. El-Nasr, "The art of drafting: a team-oriented hero recommendation system for multiplayer online battle arena games," in 12th ACM Conference on Recommender Systems, 2018.

[9] V. Do Nascimento Silva and L. Chaimowicz, "A tutor agent for moba games," in XIV Brazilian Symposium on Computer Games and Digital Entertainment, 2015.

[10] V. Do Nascimento Silva and L. Chaimowicz, "On the development of intelligent agents for moba games," in 14th Brazilian Symposium on Computer Games and
Digital Entertainment, 2015.

[11] M. P. Silva, V. Do Nascimento Silva and L. Chaimowicz, "Dynamic difficulty adjustment on MOBA games," Entertainment Computing, vol. 18, pp. 103-123, 2017.

[12] M. Z. A. Z. Aznin, N. M. Diah and N. A. S. Abdullah, "Expert system for dota 2 character selection using rule-based technique," in International Visual Informatics Conference, 2019.

[13] W. Looi, M. Dhaliwal, R. Alhajj and J. Rokne, "Recommender system for items in dota 2," IEEE Transactions on Games, vol. 11, no. 4, pp. 396-404, 2018.

[14] M. Schubert, A. Drachen and T. Mahlmann, "Esports analytics through encounter detection," in MIT Sloan Sports Analytics Conference, 2016.

[15] A. Semenov, P. Romov, S. Korolev, D. Yashkov and K. Neklyudov, "Performance of machine learning algorithms in predicting game outcome from drafts in dota 2," in International Conference on Analysis of Images, Social Networks and Texts, 2016.

[16] M. Aung, V. Bonometti, A. Drachen, P. Cowling, A. V. Kokkinakis, C. Yoder and A. Wade, "Predicting skill learning in a large, longitudinal moba dataset," in IEEE Conference on Computational Intelligence and Games (CIG), 2018.

[17] I. Porokhnenko, P. Polezhaev and A. Shukhman, "Machine learning approaches to choose heroes in dota 2," in 24th Conference of Open Innovations Association (FRUCT), 2019.

[18] A. Drachen, M. Yancey, J. Maguire, D. Chu, I. Y. Wang, T. Mahlmann, M. Schubert and D. Klabajan, "Skill-based differences in spatio-temporal team behaviour in defence of the ancients 2 (dota 2)," in IEEE Games Media Entertainment, 2014.

[19] R. Smit, "A machine learning approach for recommending items in league of legends," 2019.

[20] A. R. Novak, K. J. Bennett, M. A. Pluss and J. Fransen, "Performance analysis in esports: modelling performance at the 2018 League of Legends World Championship," International Journal of Sports Science \& Coaching, vol. 15, no. 5-6, pp. 809-817, 2020.

[21] X. Bang, W. Huiwen and R. Zhou, "What contributes to success in MOBA games? An empirical study of Defense of the Ancients 2," Games and Culture, vol. 14, no. 5, pp. 498-522, 2019.

[22] W. Michael and M. Deshen, "An analysis of artificial intelligence techniques in multiplayer online battle arena game environments," in Annual Conference of the South African Institute of Computer Scientists and Information Technologists, 2016.

[23] J. Eichelbaum, H. Roony and H. Olaf, "Classification of Icon Type and Cooldown State in Video Game Replays," in International Conference Image Analysis and Recognition, 2018.

[24] R. Ani, V. Harikumar, A. K. Devan and O. S. Deepa, "Victory 
prediction in League of Legends using Feature Selection and Ensemble methods," in International Conference on Intelligent Computing and Contro/ Systems (ICCS), 2019.

[25] S.-K. Lee, S.-J. Hong and S.-I. Yang, "Predicting Game Outcome in Multiplayer Online Battle Arena Games," in International Conference on Information and Communication Technology Convergence (ICTC), 2020.

[26] S.-J. Hong, S.-K. Lee and S.-I. Yang, "Champion Recommendation System of League of Legends," in International Conference on Information and Communication Technology Convergence (ICTC), 2020.

[27] L. Hanke and L. Chaimowicz, "A recommender system for hero line-ups in MOBA games," in $A A A$ / Conference on Artificial Intelligence and Interactive Digital Entertainment, 2017.

[28] L. Yu, D. Zhang, X. Chen and X. Xie, "Moba-slice: A time slice based evaluation framework of relative advantage between teams in moba games," in Workshop on Computer Games, 2018.

[29] S. Kim, D. Kim, H. G. Ahn and B. Ahn, "Implementation of user playstyle coaching using video processing and statistical methods in league of legends," Multimedia Tools and Applications, pp. 1-13, 2020.

[30] A. Summerville, M. Cook and B. Steenhuisen, "Draftanalysis of the ancients: predicting draft picks in dota 2 using machine learning," in AAA/ Conference on Artificial Intelligence and Interactive Digital Entertainment, 2016.

[31] X. Lan, L. Duan, W. Chen, R. Qin, T. Nummenmaa and J. Nummenmaa, "A player behavior model for predicting win-loss outcome in MOBA games," in nternational Conference on Advanced Data Mining and Applications, 2018.

[32] P. Goyal, A. Sapienza and E. Ferrara, "Recommending teammates with deep neural networks.," in 29th on Hypertext and Social Media, 2018.

[33] A. Katona, R. Spick, V. J. Hodge, S. Demediuk, F. Block, A. Drachen and J. A. Walker, "Time to die: Death prediction in dota 2 using deep learning," in IEEE Conference on Games (CoG), 2019.

[34] D. Ye, G. Chen, P. Zhao, F. Qiu, B. Yuan, W. Zhang, S. Chen, M. Sun, X. Li, S. Li, J. Liang, Z. Lian, B. Shi, L. Wang, T. Shi, Q. Fu, W. Yang and L. Huang, "Supervised Learning Achieves Human-Level Performance in MOBA Games: A Case Study of Honor of Kings," IEEE Transactions on Neural Networks and Learning Systems, pp. 1-11, 2020.

[35] A. Villa, V. Araujo, F. Cattan and D. Parra, "Interpretable Contextual Team-aware Item Recommendation: Application in Multiplayer Online Battle Arena Games," in Fourteenth ACM Conference on Recommender Systems, 2020.

[36] Q. Li, P. Xu, Y. Y. Chan, Y. Wang, Z. Wang, H. Qu and X. Ma, "A visual analytics approach for understanding reasons behind snowballing and comeback in moba games," IEEE Transactions on Visualization and Computer Graphics, vol. 23, no. 1, pp. 211-220, 2017.
[37] E. Carlini and A. Lulli, "A spatial analysis of multiplayer online battle arena mobility traces," in European Conference on Parallel Processing.

[38] A. Sapienza, H. Peng and E. Ferrara, "Performance dynamics and success in online games," in IEEE International Conference on Data Mining Workshops (ICDMW).

[39] F. Block, V. Hodge, S. Hobson, N. Septhon, S. Devlin, M. F. Ursu, A. Drachen and P. I. Cowling, "Narrative bytes: Datadriven content production in esports.," in ACM international conference on interactive experiences for TV and online video, 2018.

[40] T. Gonçalves, P. Vieria, A. P. Alfonso, M. B. Carmo and T. Moucho, "Analysing player performance with animated maps," in 22nd international conference information visualisation (IV), 2018.

[41] A. P. Afonso, M. B. Carmo and T. Moucho, "Comparison of Visualization Tools for Matches Analysis of a MOBA Game," in 23rd International Conference Information Visualisation (IV), 2019.

[42] S. Ahmad, A. Bryant, E. Kleinman, Z. Teng, T.-H. D. Nguyen and M. S. El-Nasr, "Modeling Individual and Team Behavior through Spatio-temporal Analysis," in Annual Symposium on Computer-Human Interaction in Play, 2019.

[43] M. Anshori, F. Mar'i, M. W. Alauddin and F. A. Bachtiar, "Prediction result of dota 2 games using improved svm classifier based on particle swarm optimization," in International Conference on Sustainable Information Engineering and Technology (SIET), 2018.

[44] H. Lie, D. Lukas, J. Liebig and R. Nayak, "A Novel Learningto-Rank Method for Automated Camera Movement Control in E-Sports Spectating," in Australasian Conference on Data Mining, Singapore, 2018.

[45] F. F. do Nascimento, A. S. da Costa Melo, I. B. da Costa and L. B. Marinho, "Profiling successful team behaviors in League of Legends," in 23rd Brazillian Symposium on Multimedia and the Web, 2017.

[46] S. Demediuk, A. Murrin, D. Bulger, M. Hitchens, A. Drachen, W. L. Raffe and M. Tamassia, "Player retention in league of legends: a study using survival analysis," in Australasian Computer Science Week Multiconference, 2018.

[47] S. A. Halim, Y. Indrianti, Y. Udjaja, J. Moniaga and B. A. Makalew, "The Repercussions of Game Multiplayer Online Battle Arena," in International Conference of Artificial Intelligence and Information Technology (ICA/IT), 2019.

[48] E. Kleinman, S. Ahmad, Z. Teng, A. Bryant, T.-H. D. Nguyen, C. Harteveld and M. S. El-Nasr, "'And then they died": Using Action Sequences for Data Driven, Context Aware Gameplay Analysis," in International Conference on the Foundations of Digital Games, 2020.

[49] L. M. Costa, A. C. C. Souza and F. C. M. Souza, "An approach for team composition in league of legends using genetic algorithm," in 18th Brazilian Symposium on 
Computer Games and Digital Entertainment (SBGames), 2019.

[50] T. Tsogbadrakh and M. Spradling, "Genetic Approach to Stable Partitioning in Online Role Based Hedonic Games," in IEEE Congress on Evolutionary Computation (CEC), 2019.

[51] M. Mora-Cantallops and M.-Á. Sicilia, "Player-centric networks in League of Legends," Social Networks, vol. 55, pp. 149-159, 2018.

[52] M. Mora-Cantallops and M.-Á. Sicilia, "Team efficiency and network structure: The case of professional League of Legends," Social Networks, vol. 58, pp. 105-115, 2019.

[53] C. Ringer, J. A. Walker and M. Nicolaou, "Multimodal joint emotion and game context recognition in league of legends livestreams," in IEEE Conference on Games (COG), 2019.

[54] D.-H. Kim, C. Lee and K.-S. Chung, "A confidencecalibrated moba game winner predictor," in IEEE Conference on Games (CoG), 2020.

[55] O. Cavadenti, V. Codocedo, J.-F. Boulicaut and M. Kaytoue, "What did i do wrong in my MOBA game? Mining patterns discriminating deviant behaviours," in IEEE International Conference on Data Science and Advanced Analytics (DSAA), 2016.

[56] N. P. H. Pratama, S. M. S. Nugroho and E. M. Yuniarno, "Fuzzy controller based Al for dynamic difficulty adjustment for defense of the Ancient 2 (DotA2)," in Fuzzy controller based Al for dynamic difficulty adjustment for defense of the Ancient 2 (DotA2), 2016.

[57] M. Waltham and D. Moodley, "An analysis of artificial intelligence techniques in multiplayer online battle arena game environments," in Annual Conference of the South African Institute of Computer Scientists and Information Technologists, 2016.

[58] T. D. Do, D. S. Yu, S. Anwer and S. I. Wang, "Using Collaborative Filtering to Recommend Champions in League of Legends," in IEEE Conference on Games (COG), 2020.

[59] Z. Cleghern, S. Lahiri, O. Özaltin and D. L. Roberts, "Predicting future states in dota 2 using value-split models of time series attribute data," in 12th International Conference on the Foundations of Digital Games, 2017.

[60] I. Makarov, D. Savostyanov, B. Litvyakov and D. I. Ignatov, "Predicting winning team and probabilistic ratings in "Dota 2" and "Counter-Strike: Global Offensive" video games.", in International Conference on Analysis of Images, Social Networks and Texts, Cham, 2017.

[61] M. Hirth, K. Borchert, F. Allendorf, F. Metzger and T. Hoßfeld, "Crowd-based Study of Gameplay Impairments and Player Performance in DOTA 2," in 4th Internet-QOE Workshop on QoE-based Analysis and Management of Data Communication Networks, 2019.

[62] L. Wang, Y. Tang and J. Liu, "WPQA: A Gaming Support System Based on Machine Learning and Knowledge
Graph," in Joint International Semantic Technology Conference, Singapore, 2019.

[63] D. Gordeau and L. Archambault, "Discriminative neural network for hero selection in professional Heroes of the Storm and DOTA 2," IEEE Transactions on Games, 2020.

[64] L. Zhang, C. Xu, Y. Gao, Y. Han, X. Du and Z. Tian, "Improved Dota2 lineup recommendation model based on a bidirectional LSTM," Tsinghua Science and Technology, vol. 25, no. 6, pp. 712-720, 2020.

[65] N. Wang, L. Li, L. Xiao, G. Yang and Y. Zhou, "Outcome prediction of dota2 using machine learning methods," in International Conference on Mathematics and Artificial Intelligence, 2018.

[66] B. Xia, H. Wang and R. Zhou, "What contributes to success in MOBA games? An empirical study of Defense of the Ancients 2," Games and Culture, vol. 14, no. 5, pp. 498522, 2019.

[67] J. G. Reitman, "Distributed cognition and temporal knowledge in league of legends," International Journal of Gaming and Computer-Mediated Simulations (IJGCMS), vol. 10, no. 1, pp. 23-41, 2018.

[68] Z. Chen, Y. Yang, C. Tan, D. Cheng, A. Chen and Y. Zhuang, "What makes a good team? A large-scale study on the effect of team composition in Honor of Kings," in The World Wide Web Conference, 2019.

[69] V. Araujo, F. Rios and D. Parra, "Data mining for item recommendation in MOBA games," in 13th ACM Conference on Recommender Systems, 2019.

[70] A. Kica, A. La Manna, L. O'Donell, T. Paolillo and M. Claypool, "Nerfs, Buffs and Bugs - Analysis of the Impact of Patching on League of Legends," in International Conference on Collaboration Technologies and Systems (CTS), 2016. 
